\title{
Immunological and biochemical characterization and classification of mycobacterial antigens
}

\author{
M Harboe \& H G Wiker \\ University of Oslo, Institute of Immunology and Rheumatology, \\ Fr. Qvamsgt. 1, N-0172 Oslo 1, Norway
}

The mycobacterial cell has a very complex structure and contains a multitude of proteins that are immunogenic during hyperimmunization of experimental animals. A major part of this complexity is reflected in the $\mathrm{B}$ cell response resulting in antibody production during mycobacterial infections in humans.

Using Mycobacterium bovis BCG as a model system, the complexity of the protein content may be directly demonstrated by twodimentional polyacrylamide gel electrophoresis (2D-PAGE) of concentrated culture fluid after growth on the wholly synthetic Sauton medium. The obtained pattern shows a multitude of spots of different size and intensity (Figure 1).

That the complexity of the immunogenic components of the mycobacterial cell which induce $\mathrm{B}$ cell responses is of a similar order is directly demonstrable by crossed immunoelectrophoresis (CIE), which is an immunological method based on the use of precipitating antibodies with great resolving power and ability to demonstrate and identify individual immunogenic components in the mycobacterial cell and culture fluids (Figure 2). In work with mycobacterial antigens, it is thus evident that proper identification of the reactive components is an essential requirement. At least in our hands, the antigenic content in armadillogrown Mycobacterium leprae is less complex (1), and the number of different proteins in $M$. leprae reacting with the monoclonal antibodies available so far (2) is also considerably lower.

We have used $M$. bovis $\mathrm{BCG}$ and Mycobacterium tuberculosis as model systems to study the reactivity of various mycobacterial antigens and to develop systems allowing identification of individual antigens in mycobacteria in a way permitting standardization and consistent identification of individual components in different laboratories (3).

2D-PAGE followed by silver staining shows the different protein spots, but further studies are needed to define the structural relationship between protein components forming the different spots.

Quite often, several spots are located in a row due to the presence of distinct protein entities with different isoelectric point but with similar, and in several cases, identical molecular weight. The spots termed MPB70 and MPB80 in the 2D-PAGE pattern of culture fluid after growth of the Tokyo substrain of $M$. bovis BCG on Sauton medium illustrate this point (Figure 1). They are both $18 \mathrm{~K}$ proteins, but with different isoelectric points. Both have been purified to homogeneity by S. Nagai et al. $(4,5)$ as clearly distinct proteins. Immunologically, they have been indistinguishable so far. The $\mathrm{N}$-terminal amino acid sequence is identical 


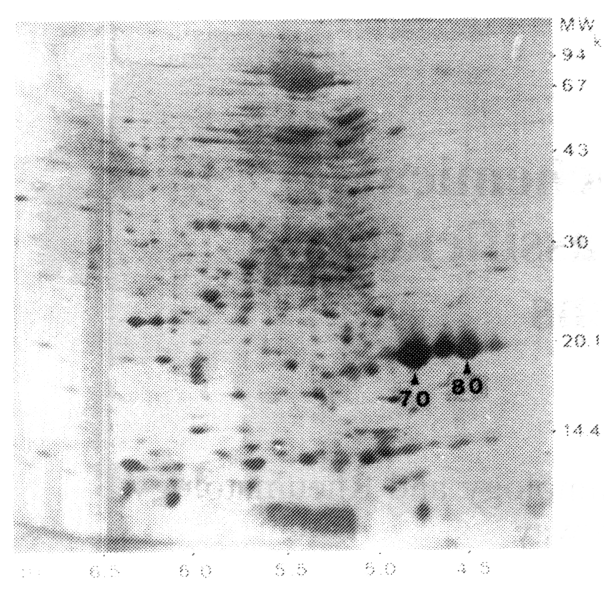

Anti-BCG

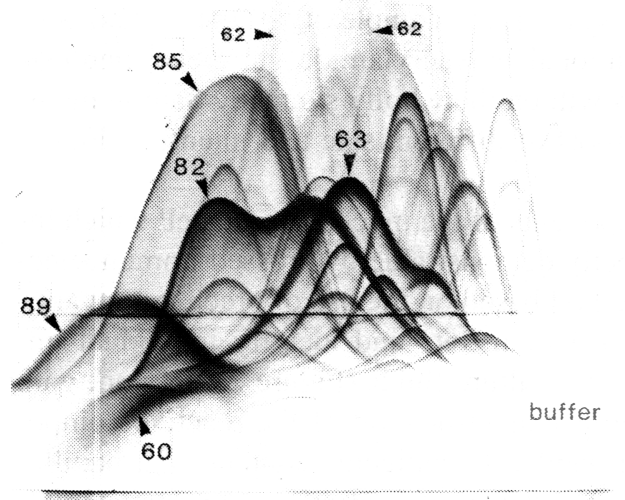

)$_{B C G}$

culture fluid
Figure 1

Two-dimentional PAGE pattern of BCG Tokyo culture fluid. After being stained with Coomassie brilliant blue, the slab was submitted to silver staining. Molecular weight $(M W)$, expressed in kilodalton, is indicated along the vertical axis and isolectric point (pI) is indicated along the horizontal axis. The arrows and numbers indicate the positions of MPB70 and MPB80.

\section{Figure 2}

CIE of M. bovis Copenhagen culture fluid. The antigens were precipitated by polyvalant immunoglobulin against BCG Copenhagen applied in the top gel. The plate was stained with Coomassie brilliant blue. Some major precipitates are indicated with arrows and numbers.

for 30 residues. 2D-PAGE of BCG Tokyo culture fluid followed by blotting and exposure to antisera made against MPB70 constantly gave 3 spots, corresponding to the silver stained MPB70 and MPB80 spots and a third major spot being situated between those two. These three proteins probably represent closely similar forms of the same gene product exposed to postsynthetic changes in the charge of a few amino acid residues. These proteins show a very high species specificity $(6,7)$ which is rare at component level in mycobacteria. They also show the marked influence of regulatory mechanisms since they occur in markedly different concentrations, at least 100-fold, in different substrains of BCG (7). 
The BCG85 antigen complex (8) illustrates that components with different isoelectric point and molecular weight may show typical reactions of partial identity. This is rare, and we have only seen this phenomenon in BCG in this complex and with one additional major component in CIE, namely BCG antigen 63.

It is also possible that two highly crossreactive antigens may form two isolated precipitates. This may be the case if the antigens are widely separated during the first dimension electrophoresis. We do not have any examples of such a phenomenon, but BCG antigen 82 sometimes presents with a double peaked precipitate consisting of two antigens partially separated during first dimention electrophoresis (Figure 2).

The BCG85B component is a $29 \mathrm{~K}$ protein whose spot in the 2D-PAGE pattern has been identified, while the BCG85A component gives a series of spots in the $31-32 \mathrm{~K}$ area corresponding to several constituents of identical molecular weight with different isoelectric points. The BCG85 complex gives a very characteristic pattern by CIE of BCG culture fluids, the appearance depending greatly on the properties of the antiserum. Some antisera distinguish between the components of the complex very nicely. In other instances, a single precipitate involving the whole complex is seen (8). Incorporation of hydroxyapatite in the intermediate gel of CIE plates with BCG culture fluid in the circular antigen well and polyvalent anti-BCG immunoglobulin in the upper gel gives a very striking pattern with only one main precipitate appearing in the top gel since all other antigenic constituents are firmly bound to the hydroxyapatite in the intermediate gel with the buffer system used. This CIE pattern immediately suggested that hydroxyapatite chromatography should be a useful principle for purification of proteins constituting the BCG85 complex, as indeed proved to be the case (8).

One precipitate in CIE. One protein?

In our experience, this is the case in most, but not all instances. Theoretical analyses show, however, that proteins consisting of several distinct polypeptide chains, and distinct proteins forming a complex, give a single precipitate in CIE following reaction with antibodies against the different constituents.

The $M$. leprae line 7 corresponds to a cell wall associated macromolecular complex containing carbohydrate, lipid and polypeptide moieties $(9,10)$. It contains the major $65 \mathrm{~K}$ and $36 \mathrm{~K}$ proteins of $M$. leprae (2).

Line 6, which in some instances is formed quite distinctly from line 7 and in other instances appear to fuse with line 7, corresponds to free polysaccharide antigen (arabinomannan + arabinogalactan).

Line 2 corresponds to a $35 \mathrm{~K}$ protein reacting with the monoclonal antibody ML 04-A1 produced by $\mathrm{J}$. Ivanyi (11).

$M$. leprae antigen 11 (12) corresponds to a $12 \mathrm{~K}$ protein reacting with the monoclonal antibody ML06 against an M. leprae specific epitope (11).

The monoclonal antibody $\mathrm{L} 7$ produced by $\mathrm{W}$. J. Britton against a $70 \mathrm{~K}$ protein present in $M$. bovis $\mathrm{BCG}$ reacts in Western blotting experiments with a $70 \mathrm{~K}$ protein in $M$. bovis $\mathrm{BCG}$, $M$. leprae and several other mycobacterial species (13). When this labelled monoclonal antibody was incorporated together with polyvalent anti- $M$. leprae in the top gel of CIE plates with $M$. leprae sonicate in the circular antigen well and the plates were washed, dried and subjected to autoradiography, radioactivity in the film appeared in a broad diffuse band in the top gel which did not correspond to any of the precipitates appearing after protein staining of the plate. The corresponding antigen in $M$. leprae is thus poorly detected by our present CIE $M$. leprae/anti-M. leprae systems. Similar experiments performed with the BCG system showed that this clone reacts intensely with the BCG antigen 63 which is a major antigenic component in the BCG pattern (Figure 2). 
Molecular weight is extensively used as criterion for identification of antigens in mycobacteria, other bacteria and virues, and with good reasons. It needs to be used, however, with caution since distinct proteins may have similar molecular weights as the 2D-PAGE pattern shows (Figure 1) not permitting distinction by one-dimentional SDS-PAGE.

The most anodic precipitating component, forming line 10 in CIE plates is a very characteristic component in $M$. tuberculosis. It is strongly immunogenic, forms a distinct peak in M. tuberculosis patterns, but is barely detectable in several strains of BCG. It is due to reaction with a $43 \mathrm{~K}$ molecule, apparently being labile with different $\mathrm{K}$ values in different $M$. $t u$ berculosis antigen preparations, while the precipitate in CIE allows definite identification.

In several instances enzyme activity is a precise indicator of the nature of precipitating components and should permit definite identification of individual components in the pattern in different laboratories, even with different antisera in the top gel giving marked variations in the overall precipitate patterns. Superoxide dismutase (SOD) activity is present in $M$. leprae antigen 4 , BCG antigen 62 , in the corresponding antigen in $M$. tuberculosis, and in the cross-reacting $M$. lepraemurium antigen 13. Catalase is in BCG antigen 56 (Closs, personal communication), and esterase activity appears in the BCG antigen 60 line (Harboe, unpublished observations).

In general, reagents or procedures which affect a single component or a few components are particularly suitable for identification of individual components in the complex CIE patterns. Monoclonal antibodies are obvious examples of reagents reacting with single components detectable by autoradiography (14) or by reaction with enzyme labelled secondary antibodies (15). By immunization with precipitates cut out of CIE plates, polyclonal, precipitating antibodies reacting with single components of mycobacteria can be reliably produced (16). These are also very valuable reagents for identification of individual, selected components in culture fluids and sonicates of mycobacteria.

In experiments studying the reactivity of Concanavalin A (Con A) incorporating the lectin in the intermediate gel in regular plates and in control plates containing EDTA in the buffer to block the Con A reactivity, it was detected that binding of divalent cations by EDTA resulted in disappearance of a single component from the top gel. The BCG antigen 8 precipitate consistently disappeared. Similar observations have been made with regard to $M$. $t u$ berculosis antigen 8 (Wiker unpublished observations). Con A reacts with BCG antigen 60, 89 , and 78, and the corresponding antigens of $M$. tuberculosis. It should be noted that BCG antigen 78 , and the corresponding line in the $M$. tuberculosis system, correspond to $M$. $t u$ berculosis «antigen 5» extensively studied by Daniel et al. (17) based on the US-Japan Immunoelectrophoresis Reference System for antigens of M. tuberculosis (18).

We conclude that a combination of biochemical techniques and studies of the reactivity with polyvalent, precipitating antibodies in systems providing high resolving power like crossed immunoelectrophoresis present powerful tools for reliable identification of individual constituents of mycobacteria before further detailed studies of their immunological reactivity at epitope level towards $\mathrm{B}$ and $\mathrm{T}$ cells.

\section{References}

1 Harboe M, Closs O, Bjorvatn B, Kronvall G, Axelsen N H. Antibody response in rabbits to immunization with Mycobacterium leprae. Infect Immun, 1977; 18: 792-805.

2 Engers H D et al. workshop. Results of a World Health Organization-sponsored workshop to characterize antigens recognized by mycobacterium-specific monoclonal antibodies. Infect Immun, 1985; 51: 718-20. 
3 Closs O, Harboe M, Axelsen N H, Bunch-Christensen K, Magnusson M. The antigens of Mycobacterium bovis, strain BCG, studied by crossed immunoelectrophoresis: a reference system. Scand J Immunol, 1980; 12: 249-63.

4 Harboe M, Nagai S, Patarroyo M E, Lucia Torres M, Ramirez C, Cruz M. Properties of the MPB64, MPB70 and MPB80 proteins of Mycobacterium bovis BCG. Infect Immun, 1986; 52: 293-302.

5 Nagai S, Matsumoto J, Nagasuga T. Specific skin-reactive protein from culture filtrate of Mycobacterium bovis BCG. Infect Immun, 1981; 31: 1152-60.

6 Miura K, Nagai S, Kinomoto M, Haga S, Tokunaga T. Comparative studies of various substrains of Mycobacterium bovis BCG on the production of an antigenic protein, MPB70. Infect Immun, 1983; 39: 540-45.

7 Harboe M, Nagai S. MPB70, a unique antigen of Mycobacterium bovis BCG. Am Rev Respir Dis, 1984; 129: 444-52.

8 Wiker H G, Harboe M, Lea T. Purification and characterization of two protein antigens from the heterogenous BCG85 complex in Mycobacterium bovis, BCG. Int Archs Allergy Appl Immun, 1986; In press.

9 Harboe M, Closs O, Reitan L J, Draper P. Demonstration of antibodies reacting with different determinants on Mycobacterium leprae antigen 7. Int J leprosy, 1981; 49: 147-58.

10 Reitan L J, Closs O, Jantzen E. Further characterization including preliminary chemical analysis of antigen MLWl from Mycobacterium leprae. Int Archs Allergy Appl Immun, 1985; 78: 269-76.

11 Ivanyi J, Sinha S, Aston R, Cussell D, Keen M, Sengupta U. Definition of species specific and cross-reactive antigenic determinants of Mycobacterium leprae using monoclonal antibodies. Clin Exp Immunol, 1983; 52: 528-36.

12 Harboe M. The immunology of leprosy. Chapter 4 in Leprosy. R. C. Hastings, Ed. Churchill Livingstone, Edinburgh 1985. 331 pp.

13 Britton W J, Hellqvist L, Basten A, Raison R L. Mycobacterium leprae antigens involved in human immune responses. J Immunol, 1985; 135: 4171-77.

14 Harboe M, Coates A R M, Hewitt J. Characterization of the specificity of monoclonal antibodies against Mycobacterium tuberculosis by crossed immunoelectrophoresis. Scand J Immunol, 1985; 22: 93-8.

15 Skjødt K, Schou C, Koch C. Assay for the specificity of monoclonal antibodies in crossed immunoelectrophoresis. J Immunol Meth, 1984; 72: 243-49.

16 Harboe M, Closs O, Deverill J. Production of monospecific antisera against antigenic components of Mycobacterium bovis (BCG). Scand J Immunol, 1976; 5: 861-66.

17 Daniel T M, Anderson P A. The isolation by immunosorbent affinity chromatography and physicochemical characterization of Mycobacterium tuberculosis antigen 5. Am Rev Respir Dis, 1978; 117: 533-39.

18 Janicki B W, Chaparas S D, Daniel T M, Kubica G P, Wright jr G L, Yee G S. A reference system for antigens of Mycobacterium tuberculosis. Am Rev Respir Dis, 1971; 104: $602-4$. 